\title{
Editorial
}

\section{Reducing Violence Against Doctors and Healthcare Workers: Financing for Better Infrastructure}

\author{
Rajeev Gupta \\ ${ }^{I}$ Chair, Academic and Research Development Unit, Editor, RUHS Journal of Health Sciences, Rajasthan \\ University of Health Sciences, ${ }^{2}$ Department of Medicine, Eternal Heart Care Centre and Research Institute, \\ Jaipur, Rajasthan, India
}

Workplace violence against physicians and other healthcare professionals in India is in the news. ${ }^{1}$ The US National Institute for Occupational Safety and Health defines workplace violence as "violent acts (including physical assaults and threats of assaults) directed toward persons at work or on duty". The US Department of Labor defines workplace violence as an action (verbal, written, or physical aggression) that is intended to control or cause, or is capable of causing, death or serious bodily injury to oneself or others, or damage to property. Workplace violence includes abusive behavior toward authority, intimidating or harassing behavior, and threats. ${ }^{2}$ Between $8 \%$ to $38 \%$ of health workers suffer physical violence at some point in their careers. ${ }^{3}$

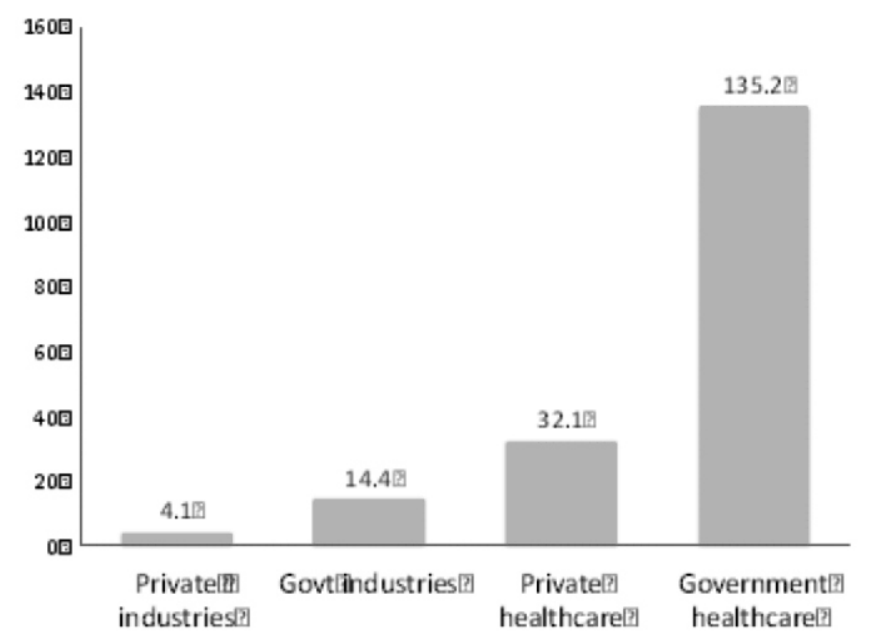

Figure: Rates of workplace violence against healthcare workers in USA requiring missed workdays.

The exact extent of such violence is unknown in our country and the incidences reported so far may be a tip of the iceberg. Workplace violence has been classified into four types; (a) perpetrator has no association with the workplace or employees, (b) perpetrator is a customer or patient of the workplace or employees, (c) perpetrator is a current or former employee of the workplace, and (d) perpetrator has a workplace employees, none with the workplace. ${ }^{4}$ In USA, a high incidence of workplace violence has been reported. Rates of workplace violence with injury requiring missed workdays has been studied in US industries. In the year 2014, rate per 10,000 workers was 4.1 for all private industries, 14.4 for all state industries, 32.1 for private healthcare and social services and 135.2 for state healthcare and social services (Figure). It has been reported that there are large variations in reporting of data to various national agencies and minor forms of violence (verbal assaults threats, physical assault and battery) not requiring absenteeism may be 10 times more common. Workplace violence is also present in nonhospital settings such as pre-hospital settings, primary care and other office-based practices, and home healthcare and can be against nurses, physicians, other healthcare workers and administrators. ${ }^{3}$ Similar data are not available for India. ${ }^{5}$ The focus in India has been only on doctors and not the whole category of healthcare professionals.

The acts of such violence in India may be reflective of general unease in the society, strain of reforms and rapid pace of societal change, social instability, poor image of physicians, poor quality of healthcare systems, vulnerability of small and medium healthcare establishments, lack of faith in judicial process, mob mentality, low health literacy, poor communication, lack of security and cost of healthcare. ${ }^{5}$ The negative consequences of violence impacts the delivery of healthcare services, which could further lead to deterioration in the quality of care 
provided and the decisionby health workers, including physicians, to leave the health care professionals. This in turn can result in a reduction in health services available to the general population, and an increase in health costs. ${ }^{3}$

Interventions: Health work place interventions have been divided into pre-emptive, organizational, environmental, individual-focused and after-the-event interventions by International Labour Organization and others. ${ }^{3}$ Legis-lation for harsher punishment for violence against healthcare workers has been used widely, also in India., At the hospital level the preventive program should be based on issues involving individual workers, lawenforcement officials and healthcare organizations. Strategies include prompt reporting of events, training in aggression de-escalation techniques, training in selfdefence and creating infrastructure (fences, security cameras, metal detectors, guards, etc). Important are improving staffing levels during busy periods to reduce crowding and waiting times, decrease worker turnover and provide adequate security and mental health professionals on site. ${ }^{3}$ Supervisor support is important.

Legislative solutions to control violence against healthcare workers have been proposed in India. The Prevention of Violence Against Medicare Persons and Institutions Act has been notified across the country but has failed to address the issue. Societal actions are needed which involve better health and general literacy in populations and improved communication skills among physicians and health workers. ${ }^{6}$ For health providers it is important to realize the limits of medicine, importance of informed consent, proper documentation of patient's course of illness and improve communication skills (art of medicine).

Organizational interventions should be the most important priority. ${ }^{7}$ Better infrastructure and staffing are crucial. This shall need enhanced government spending on healthcare. In countries where the healthcare is free at the point of care, e.g. UK, Western Europe, the acts of violence are less common. ${ }^{7}$ Adequate financing would improve organizational infrastructure with better physical environment, workplace design (access, space, waiting areas, fixtures and fittings, alarm systems and surveillance cameras and the premises), and promote adequate staffing, better management, better information and communication and general work practices.

Financial issues are one of the most important stimulants for health place violence in India. ${ }^{3}$ Greater financing for health infrastructure and creating job opportunities for paramedical workers with adequate remuneration are crucial. The focus so far has ben on physician shortage. However, for creating high quality health systems where incidence of violence is low, investment in infrastructure (buildings, equipments) and man-power is essential. ${ }^{8}$ One physician needs a support staff of dozens of healthworkers. Focus on high-quality and free universal healthcare as envisaged in the recent national health policies and time-bound proposals of increased funding by the government are steps in the right direction.

\section{REFERENCES}

1. Bhagat C. Don't make doctors the fall guy for India's poor healthcare. Times of India (Jaipur). 23 Jun 2019; 12.

2. Patient Safety Advisory Group: Joint Commission. Physical and verbal violence against health care workers. Sentinel Event Alert. 2018; 59:1-9.

3. Joint Programme on Workplace Violence in Health Sector. Framework guidelines for addressing workplace violence in the health sector. Geneva. International Labour Organization, International Council of Nurses, World Health Organization and Public Services International. 2002. Available at: https://www.who.int/violence_injury _prevention/violence/interpersonal/en/WVguidelinesEN .pdf?ua=1\&ua=1. Accessed 23 June 2019.

4. Phillips JP. Workplace violence against healthcare workers in the United States. N Engl J Med. 2016; 374:1661-69.

5. Nagpal N. Incidence of violence against doctors in India: can these be prevented. Natl Med J India. 2017; 30:97-100.

6. Gupta R. Lost in numbers: the art and science of medicine. RUHS J Health Sciences. 2018; 3:59-60.

7. Cooper CL, Swanson N. Workplace violence in the health sector: state of the art. Available at: https://www.who.int/ violence_injury_prevention/violence/activities/workpla ce/WVstateart.pdf?ua=1. Accessed 23 June 2019.

8. Gupta R, Khedar RS, Gupta N, Chaudhary AK. Lack of physicians is not the root cause for poor rural healthcare in India. BMJ. 2014; 348:g54. Response. 